PROCEEDINGS OF THE

AMERICAN MATHEMATICAL SOCIETY

Volume 132, Number 4, Pages 1095-1102

S 0002-9939(03)07246-0

Article electronically published on September 18, 2003

\title{
A REMARK ON GLOBAL EXISTENCE FOR SMALL INITIAL DATA \\ OF THE MINIMAL SURFACE EQUATION IN MINKOWSKIAN SPACE TIME
}

\author{
HANS LINDBLAD
}

(Communicated by David S. Tartakoff)

\begin{abstract}
We show that the nonlinear wave equation corresponding to the minimal surface equation in Minkowski space time has a global solution for sufficiently small initial data.
\end{abstract}

\section{INTRODUCTION}

We show that the nonlinear wave equation corresponding to the minimal surface equation in Minkowski space time

$$
\frac{\partial}{\partial t} \frac{\phi_{t}}{\sqrt{1+\left|\nabla_{x} \phi\right|^{2}-\phi_{t}^{2}}}-\sum_{i=1}^{n} \frac{\partial}{\partial x^{i}} \frac{\phi_{i}}{\sqrt{1+\left|\nabla_{x} \phi\right|^{2}-\phi_{t}^{2}}}=0
$$

where $\phi_{i}=\partial \phi / \partial x^{i}, \phi_{t}=\partial \phi / \partial t$, has global solutions for sufficiently small initial data

$$
\left.\phi\right|_{t=0}=\varepsilon f,\left.\quad \phi_{t}\right|_{t=0}=\varepsilon g,
$$

$f \in C_{0}^{\infty}$ and $g \in C_{0}^{\infty}$, i.e., (1.1)-(1.2) has for fixed $f$ and $g$ a solution for all $t \geq 0$ if $\varepsilon>0$ is sufficiently small. This is an interesting model in Lorentzian geometry proposed to me by Hamilton [Ha1]. It is also the equation for a membrane in field theory; see Hoppe [Ho1]. Also, Huisken and Struwe [HS1] have some recent results related to local existence for (1.1).

What makes the proof go through also in the physically important case of two space dimensions ( $\phi$ itself corresponds to the third space dimension) is that the nonlinear terms satisfy the so-called "null condition" of Christodoulou [C1] and Klainerman [K2], [K4]. The purpose of this note is to present two simple proofs making use of the extra symmetries of the equation. The first proof uses a version of the method of [K2] that works also in two space dimensions. For equations in divergence form we can get a good $L^{2}$ estimate for the solution itself (see [L1]) that replaces the conformal energy estimate used in [K2]. The second proof uses a simplified version $\mathrm{C} 2$ of the method of [C1, and it works also in one space dimension due to the fact that the equation satisfies a "double null condition"; see (1.5). The first proof does not work in the case of one space dimension but it has

Received by the editors December 9, 2002

2000 Mathematics Subject Classification. Primary 35-xx.

(C)2003 American Mathematical Society 
the advantage that it does not require compact support of initial data but merely some decay at infinity. Let

$$
\square=\partial_{t}^{2}-\sum_{i=1}^{3} \partial_{i}^{2} \quad \text { and } \quad Q_{00}(\phi, \psi)=\phi_{t} \psi_{t}-\sum_{i=1}^{n} \phi_{i} \psi_{i}
$$

be a null form. We can write (1.1) as a wave equation with a right-hand side in divergence form

$$
\square \phi=\partial_{t}\left(\phi_{t} F\left(Q_{00}(\phi, \phi)\right)\right)-\sum_{i=1}^{n} \partial_{i}\left(\phi_{i} F\left(Q_{00}(\phi, \phi)\right)\right),
$$

where $F(Q)=-1+1 / \sqrt{1-Q}$. We can alternatively write it with null forms

$$
\square \phi=-\frac{Q_{00}\left(\phi, Q_{00}(\phi, \phi)\right)}{2\left(1-Q_{00}(\phi, \phi)\right)} .
$$

The idea behind the proofs is that we expect solutions of the nonlinear wave equation to decay like solutions of the linear homogeneous wave equation $\square \phi=0$; i.e., $\left|\partial^{\alpha} \phi\right| \leq C \varepsilon(1+t)^{-(n-1) / 2}$. This will make the right-hand sides of the nonlinear wave equations small for large $t$, and hence these equations will be close to the homogeneous case; so we can close the argument. What makes the proof go through in the lower-dimensional case is that for a null form there is an additional cancellation which leads to an additional decay of a factor of $(1+t)^{-1}$ : $\left|\partial^{\alpha} Q(\phi, \phi)\right| \leq C \varepsilon^{2}(1+t)^{-(n-1)-1}$.

\section{THE PROOF USING VECTOR FIELDS}

The idea of this argument is to exploit that solutions of linear wave equations $\square v=0$ satisfy the decay estimate; see, e.g., $\mathrm{Hö2}$,

$$
|v(t, x)| \leq C(f, g) \varepsilon(1+t+|x|)^{-(n-1) / 2}(1+|t-| x||)^{-(n-1) / 2},
$$

where $C(f, g)$ is a constant depending on some weighted Sobolev norm of initial data $(f, g)$. We will use vector fields to obtain this kind of decay. For $(t, x) \in \mathbf{R}^{1+n}$ denote $\partial_{t}$ by $\partial_{0}$ and $\partial_{x_{j}}$ by $\partial_{j}$ for $j=1, \ldots, n$. Let

$$
\Gamma_{j k}=\lambda_{j} x_{j} \partial_{k}-\lambda_{k} x_{k} \partial_{j}, \quad \text { for } \quad i \neq j, \quad \text { and } \quad \Gamma_{00}=\sum_{0}^{n} x_{j} \partial_{j}
$$

where $\lambda=(1,-1, \ldots,-1)$ and $x_{0}=t . \Gamma_{j k}$, for $(j, k) \neq(0,0)$ are the vector fields associated with the Lorentz group that all commute with $\square$, and $\Gamma_{00}$ is the scaling vector fields whose commutator is $\left[\Gamma_{00}, \square\right]=-2 \square$. $\Gamma$ will symbolically stand for any of the vector fields $\Gamma_{i j}$ or $\partial_{j}, i, j=0,1, \ldots, n$ and we will write $\Gamma^{I}$ for a product of $|I|$ of such vector fields. We note that $\left[\partial_{i}, \Gamma_{j k}\right]$ is either 0 or else equal to $\pm \partial_{l}$ for some $l$. The operators $\left\{\Gamma_{j k}\right\}$ span the tangent space at every point where $t \neq|x|$. But when $t=|x|$ they only span the tangent space of the cone $t=|x|$; so we have (see [K1], [K2], [Ho1], ㅂ̈2] or [L1]

$$
|\partial \phi| \leq C(|t-| x||)^{-1} \sum\left|\Gamma_{i j} \phi\right|, \quad|\partial \phi| \leq C(t+|x|)^{-1}\left(|x|\left|\partial_{t} \phi\right|+\sum\left|\Gamma_{i j} \phi\right|\right),
$$

where $|\partial w|^{2}=\sum_{0}^{n}\left|\partial_{j} w\right|^{2}$. Now we also need to calculate the commutator of $\Gamma$ with a null form $Q$. However, in these commutators null forms other than (1.3) will 
come up. For $0 \leq i, j \leq n$ and $i \neq j$, let

$$
Q_{i j}(\phi, \psi)=\left(\partial_{i} \phi\right) \partial_{j} \psi-\left(\partial_{j} \phi\right) \partial_{i} \psi
$$

Let $Q$ symbolically stand for any of the null forms (1.3) or (2.4). Then

$$
\Gamma Q(\phi, \psi)=Q(\Gamma \phi, \psi)+Q(\phi, \Gamma \psi)+\sum a_{i j} Q_{i j}(\phi, \psi)
$$

for some constants $a_{i j}$. For a null form we have better decay close to the light cone (see [K1], [K2])

$$
|Q(\phi, \psi)| \leq C(1+|t|+|x|)^{-1}(|\partial \phi||\Gamma \psi|+|\Gamma \phi||\partial \psi|)
$$

where here $|\Gamma \phi|^{2}=\sum_{|I|=1}\left|\Gamma^{I} \phi\right|^{2}$. The decay of $\phi$ will be obtained from the fact that $\Gamma^{I} \phi$ also satisfies nonlinear wave equations of the same form. Applying $\Gamma^{I}$ to (1.4) we get

$$
\begin{array}{r}
\square \Gamma^{I} \phi=\sum_{j=0}^{n} \partial_{j}\left(\sum_{\substack{k \geq 3,\left|I_{1}\right|+\ldots+\left|I_{k}\right| \leq|I| \\
\left|I_{i}\right| \leq|I| / 2, i<k,\left|I_{k}\right| \leq|I|}} F_{j k i_{1} \ldots i_{k} I_{1} \ldots I_{k}}\left(Q_{00}(\phi, \phi)\right)\right. \\
\left.\times\left(\partial_{i_{1}} \Gamma^{I_{1}} \phi\right)\left(\partial_{i_{2}} \Gamma^{I_{2}} \phi\right) \cdots\left(\partial_{i_{k}} \Gamma^{I_{k}} \phi\right)\right) .
\end{array}
$$

Similarly, from (1.5) we get

$$
\square \Gamma^{I} \phi+\frac{Q_{00}\left(\phi, Q_{00}\left(\phi, \Gamma^{I} \phi\right)\right)}{1+Q_{00}(\phi, \phi)} \sum_{\substack{k \geq 3,\left|I_{1}\right|+\ldots+\left|I_{k}\right| \leq|I|+1,\left|I_{i}\right| \leq(|I|+1) / 2, i<k,\left|I_{k}\right| \leq|I|}} G_{k i_{1} \ldots i_{k} I_{1} \ldots I_{k}}\left(Q_{00}(\phi, \phi)\right)\left(\partial_{i_{1}} \Gamma^{I_{1}} \phi\right)
$$

and

$$
\begin{aligned}
& \square \Gamma^{I} \phi \\
& \begin{aligned}
=\sum_{k \geq 3,\left|I_{1}\right|+\ldots+\left|I_{k}\right| \leq|I|} H_{I_{1} \ldots I_{k}}^{k i_{0} \ldots i_{k}}\left(Q_{00}(\phi, \phi)\right) & Q_{i_{0} i_{1}}\left(\Gamma^{I_{1}} \phi, Q_{i_{2} j_{3}}\left(\Gamma^{I_{2}} \phi, \Gamma^{I_{3}} \phi\right)\right) \\
\times & Q_{i_{4} i_{5}}\left(\Gamma^{I_{4}} \phi, \Gamma^{I_{5}} \phi\right) \cdots Q_{i_{k-1} i_{k}}\left(\Gamma^{I_{k-1}} \phi, \Gamma^{I_{k}} \phi\right)
\end{aligned}
\end{aligned}
$$

where $k$ is odd, and if $k=3$, then this is to be interpreted as the factor $Q_{i_{4} i_{5}}\left(\Gamma^{I_{4}} \phi\right.$, $\Gamma^{I_{5}} \phi$ ) being absent.

The proof will use the energy inequality applied to derivatives of the solution and some decay estimates. The energy inequality (see [K3] or [Hö2]) for a solution of

$$
\square w+\sum_{j, k=0}^{n} \gamma^{j k}(t, x) \partial_{j} \partial_{k} w=F, \quad|\gamma|=\sum\left|\gamma^{j k}\right| \leq \frac{1}{2}
$$

says that

$$
\begin{aligned}
\|\partial w(t, \cdot)\|_{L^{2}} \leq & 2 \exp \left(\int_{0}^{t} 2\left|\gamma^{\prime}(\tau)\right| d \tau\right)\|\partial w(0, \cdot)\|_{L^{2}} \\
& +2 \int_{0}^{t} \exp \left(\int_{s}^{t} 2\left|\gamma^{\prime}(\tau)\right| d \tau\right)\|F(s, \cdot)\|_{L^{2}} d s
\end{aligned}
$$


where $\left|\gamma^{\prime}(t)\right|=\sum_{i, j, k} \sup \left|\partial_{i} \gamma^{j k}(t, \cdot)\right|$. It was not stated exactly like this in [Hö2], but (2.11) follows from the proof of the version there. As a consequence of the energy inequality, we also have the following estimate: If

$$
\square w=\sum_{0}^{n} \partial_{j} F_{j},\left.\quad w\right|_{t=0}=\varepsilon f,\left.\quad w_{t}\right|_{t=0}=\varepsilon g,
$$

then

$$
\|w(t, \cdot)\|_{L^{2}} \leq \sum_{j=0}^{n} \int_{0}^{t}\left\|F_{j}(s, \cdot)\right\|_{L^{2}} d s+C\left(f, g, F_{0}(0, \cdot)\right) m(t) \varepsilon
$$

where $m(t)=1$ if $n \geq 3, m(t)=\log (2+t)$ if $n=2$ and $C\left(f, g, F_{0}(0, \cdot)\right)$ stands for some constant depending on some weighted Sobolev norm of initial data $f$ and $g$. The proof is a trick used in [L1]; if $\square v_{j}=F_{j}$, then $\square\left(w-\sum \partial_{j} v_{j}\right)=0$. So $\|w(t, \cdot)\|_{L^{2}}$ is bounded by $\sum\left\|\partial v_{j}(t, \cdot)\right\|_{L^{2}}$, which can be estimated by the energy inequality (2.11), plus the norm for a solution of a linear homogeneous equation, which can be obtained from, e.g., (2.1). We will also need an $L^{1}-L^{\infty}$ estimate of Hörmander [Hö1] (see also Klainerman [K1], K2] for an earlier version and [L1] for a simple proof): The solution $w$ of

$$
\square w=F,\left.\quad w\right|_{t=0}=\varepsilon f,\left.\quad w_{t}\right|_{t=0}=\varepsilon g
$$

satisfies

$$
\begin{aligned}
|w(t, x)| \leq C(1+t+|x|)^{-(n-1) / 2}\left(\sum_{|I| \leq n-1} \int_{0}^{t} \|\left(\Gamma^{I} F\right)(s, \cdot) /\right. \\
\left.\quad(1+s+|\cdot|)^{(n-1) / 2} \|_{L^{1}} d s+C(f, g) \varepsilon\right) .
\end{aligned}
$$

Here the estimate for the linear homogeneous part, the second term, is (2.1). Whereas the proof of the energy inequality is merely integration by parts, the proofs of the decay estimates (2.15) and (2.1) require a detailed analysis of the fundamental solution or stationary phase.

Let $N \geq 2 n+1$ and $\delta=0$, if $n \geq 3$, and $0<\delta<1 / 2$ fixed, if $n=2$. We will now prove that

$$
\begin{aligned}
& M_{1}(t)=\sum_{|I| \leq N}\left\|\partial \Gamma^{I} \phi(t, \cdot)\right\|_{L_{2}} \leq K \varepsilon(1+t)^{\delta}, \\
& M_{2}(t)=\sum_{|I| \leq N}\left\|\Gamma^{I} \phi(t, \cdot)\right\|_{L_{2}} \leq K \varepsilon(1+t)^{\delta}, \\
& N_{1}(t)=\sum_{|J| \leq(N+1) / 2}\left\|\partial \Gamma^{J} \phi(t, \cdot)\right\|_{L^{\infty}} \leq K \varepsilon(1+t)^{-(n-1) / 2}, \\
& N_{2}(t)=\sum_{|J| \leq(N+1) / 2+1}\left\|\Gamma^{J} \phi(t, \cdot)\right\|_{L^{\infty}} \leq K \varepsilon(1+t)^{-(n-1) / 2}
\end{aligned}
$$

if $K$ is sufficiently large and $\varepsilon$ is sufficiently small. We observe that the bound for $N_{1}$ is a consequence of the bound for $N_{2}$ since, in particular, we can take one factor 
of $\Gamma=\partial$. (2.11) applied to (2.8) gives

$$
\begin{aligned}
M_{1}(t) \leq & C \varepsilon \exp \left(\int_{0}^{t} N_{1}(\tau)^{2} d \tau\right) \\
& +\int_{0}^{t} \exp \left(\int_{s}^{t} N_{1}(\tau)^{2} d \tau\right) C\left(N_{1}(s)\right) N_{1}(s)^{2} M_{1}(s) d s
\end{aligned}
$$

and (2.13) applied to (2.7) gives

$$
M_{2}(t) \leq C\left(C(f, g) m(t) \varepsilon+\int_{0}^{t} C\left(N_{1}(s)\right) N_{1}(s)^{2} M_{1}(s) d s\right) .
$$

Finally, (2.15) applied to (2.9) using (2.6) and the Cauchy-Schwartz inequality gives $(2.22)$

$$
N_{2}(t) \leq C(1+t)^{-(n-1) / 2}\left(C(f, g) \varepsilon+\int_{0}^{t} \frac{\left(N_{1}(s)+N_{2}(s)\right)}{(1+s)^{(n-1) / 2+1}}\left(M_{1}(s)+M_{2}(s)\right)^{2} d s\right)
$$

if $(N+1) / 2+1+n-1 \leq N$, i.e., $N \geq 2 n+1$. What will make the argument work also for $n=2$ is that the null condition gave an extra power of $(1+s)^{-1}$ in the integral (2.22). In fact, because we have a double null condition we actually have one more power, but we have no use of this here. The rest of the argument is now by continuity. We know that (2.16)-(2.19) are true for $t=0$ if $K$ is large enough, and we know from the local existence theorem for hyperbolic equations (see, e.g., [Hö2]) that these quantities are continuous as long as they are bounded. We now assume that $T_{1}$ is the largest number such that (2.16)-(2.19) are true for $t \leq T_{1}$ and show that these bounds together with (2.20)-(2.22) imply stronger bounds if $K$ is sufficiently large and $\varepsilon$ is sufficiently small. Hence by continuity we conclude that the bounds (2.16)-(2.19) must hold for $t \leq T_{2}$ where $T_{2}>T_{1}$, contradicting the maximality of $T_{1}$. To simplify notation, we now deal only with the most sensitive case $n=2$. Then

$$
\begin{aligned}
\exp \left(\int_{s}^{t} N_{1}(\tau)^{2} d \tau\right) & \leq \exp \left(K^{2} \varepsilon^{2} \int_{s}^{t}(1+\tau)^{-1} d \tau\right) \\
& =\exp \left(K^{2} \varepsilon^{2} \ln \left(\frac{1+t}{1+s}\right)\right)=\left(\frac{1+t}{1+s}\right)^{K^{2} \varepsilon^{2}} .
\end{aligned}
$$

So it follows from (2.20) that

$$
M_{1}(t) \leq C \varepsilon(1+t)^{K^{2} \varepsilon^{2}}+\int_{0}^{t} C \varepsilon^{3} K^{3}\left(\frac{1+t}{1+s}\right)^{K^{2} \varepsilon^{2}}(1+s)^{\delta-1} d s \leq K \varepsilon(1+t)^{\delta} / 2
$$

if $K$ is sufficiently large and $\varepsilon$ is sufficiently small. Similarly, from (2.21) we get

$$
M_{2}(t) \leq C \varepsilon \log (2+t)+\int_{0}^{t} K^{3} \varepsilon^{3}(1+s)^{\delta-1} d s \leq K \varepsilon(1+t)^{\delta} / 2
$$

if $K$ is sufficiently large and $\varepsilon$ is sufficiently small. Finally, from (2.22) we get

$$
N_{2}(t) \leq C(1+t)^{-1 / 2}\left(\varepsilon+\int_{0}^{t} K^{3} \varepsilon^{3}(1+s)^{2 \delta-2} d s\right) \leq K \varepsilon(1+t)^{-1 / 2} / 2
$$

if $K$ is sufficiently large and $\varepsilon$ is sufficiently small since $0<\delta<1 / 2$. This concludes the proof. 


\section{The PROOF USING CONFORMAL INVERSION}

We will reduce the global problem to a local problem, for which small data existence is known, using a conformal inversion or Kelvin transform. Let $\kappa: \mathbf{R}^{1+n} \ni$ $(s, y) \rightarrow(t, x) \in \mathbf{R}^{1+n}$ and $\tilde{\phi}$ be defined by

$$
\tilde{\phi}=\phi \circ \kappa \rho^{-\alpha}, \quad x^{i}=\kappa^{i}(s, y)=y^{i} / \rho, \quad \rho=s^{2}-|y|^{2}, \quad \alpha=\frac{n-1}{2},
$$

where $x^{0}=t$ and $y^{0}=s$. Let $m_{i j}=m^{i j}$ be the Minkowski metric $m_{00}=1$, $m_{i i}=-1$ if $j \geq 1$ and $m_{i j}=0$ if $i \neq j$. Then $\partial_{i} \kappa^{k}=\delta_{i}{ }^{k} / \rho-2 y^{j} y^{k} m_{i j} / \rho^{2}$. If the metric in the $x$ coordinates is $m_{i j}$, then the pull-back metric in the $y$ coordinates is given by

$$
g_{i j}=m_{k l}\left(\partial_{i} \kappa^{k}\right) \partial_{j} \kappa^{l}=m_{i j} / \rho^{2}, \quad g^{i j}=m^{i j} \rho^{2} .
$$

Then the norm is invariant: $g^{i j}\left(\partial_{i} \phi \circ \kappa\right) \partial_{j} \psi \circ \kappa=m^{i j} \phi_{i} \circ \kappa \psi_{j} \circ \kappa$ where $\partial_{i} \phi \circ \kappa=$ $\partial \phi \circ \kappa / \partial y^{i}$ and $\phi_{i}=\partial \phi / \partial x^{i}$; i.e., if $Q_{00}$ is the null form (1.3), then

$$
Q_{00}(\phi, \psi) \circ \kappa=\rho^{2} Q_{00}(\phi \circ \kappa, \psi \circ \kappa) .
$$

Expressing $\square$ in the $y$ coordinates we get

$$
\begin{aligned}
(\square \phi) \circ \kappa & =\square_{g}(\phi \circ \kappa)=(\operatorname{det} g)^{-1 / 2} \partial_{i}\left(g^{i j}(\operatorname{det} g)^{1 / 2} \partial_{j} \phi \circ \kappa\right) \\
& =\rho^{n+1} m^{i j} \partial_{i}\left(\rho^{-(n-1)} \partial_{j} \phi \circ \kappa\right) .
\end{aligned}
$$

Since the operator $\square_{g}-(n-1) R /(4 n)$, where $R$ is the scalar curvature, is conformally covariant and the scalar curvature in both cases vanishes, we obtain

$$
\square \tilde{\phi}=\rho^{-\alpha-2}(\square \phi) \circ \kappa .
$$

This also follows from (3.4) by an easy calculation using that $\square \rho^{-\alpha}=0$. Hence by $(1.5),(3.5)$ and (3.3),

$$
\square \tilde{\phi}=-\rho^{-\alpha} \frac{Q_{00}\left(\rho^{\alpha} \tilde{\phi}, \rho^{2} Q_{00}\left(\rho^{\alpha} \tilde{\phi}, \rho^{\alpha} \tilde{\phi}\right)\right)}{1-\rho^{2} Q_{00}\left(\rho^{\alpha} \tilde{\phi}, \rho^{\alpha} \tilde{\phi}\right)} .
$$

Since $m^{i j}\left(\partial_{i} \tilde{\psi}\right) \partial_{j} \rho=2\left(\Gamma_{00} \tilde{\psi}\right)$, where $\Gamma_{00}=s \partial_{s}+y^{i} \partial_{i}$ and $\Gamma_{00} \rho=2 \rho$, we obtain

$$
Q_{00}\left(\rho^{\beta} \tilde{\phi}, \rho^{\gamma} \tilde{\psi}\right)=\rho^{\beta+\gamma-1}\left(\rho Q_{00}(\tilde{\phi}, \tilde{\psi})+2 \beta \tilde{\phi}\left(\Gamma_{00} \tilde{\psi}\right)+2 \gamma \tilde{\psi}\left(\Gamma_{00} \tilde{\phi}\right)+4 \gamma \beta \tilde{\phi} \tilde{\psi}\right) .
$$

Furthermore, $\Gamma_{00} Q_{00}(\tilde{\phi}, \tilde{\phi})=2 Q_{00}\left(\tilde{\phi}, \Gamma_{00} \tilde{\phi}\right)-2 Q_{00}(\tilde{\phi}, \tilde{\phi})$. Using (3.7) twice we see that we can factor out $\rho^{3 \alpha}$ from $Q_{00}\left(\rho^{\alpha} \tilde{\phi}, \rho^{2} Q_{00}\left(\rho^{\alpha} \tilde{\phi}, \rho^{\alpha} \tilde{\phi}\right)\right)$. So (3.6) has the general form

$$
\begin{aligned}
\square \tilde{\phi}=- & \rho^{2 \alpha} \frac{\rho^{2} Q_{00}\left(\tilde{\phi}, Q_{00}(\tilde{\phi}, \tilde{\phi})\right)+\rho c_{1} \tilde{\phi} Q_{00}\left(\tilde{\phi}, \Gamma_{00} \tilde{\phi}\right)+\rho c_{2} \tilde{\phi}^{2} \Gamma_{00}^{2} \tilde{\phi}}{1-\rho^{2 \alpha}\left(\rho^{2} Q_{00}(\tilde{\phi}, \tilde{\phi})+4 \alpha \rho\left(\alpha \tilde{\phi}^{2}+\tilde{\phi} \Gamma_{00} \tilde{\phi}\right)\right)} \\
& +\rho^{2 \alpha} \frac{\rho\left(c_{3} \tilde{\phi}+c_{4} \Gamma_{00} \tilde{\phi}\right) Q_{00}(\tilde{\phi}, \tilde{\phi})+\tilde{\phi}\left(c_{5} \tilde{\phi}^{2}+c_{6} \tilde{\phi} \Gamma_{00} \tilde{\phi}+c_{7}\left(\Gamma_{00} \tilde{\phi}\right)^{2}\right)}{1-\rho^{2 \alpha}\left(\rho^{2} Q_{00}(\tilde{\phi}, \tilde{\phi})+4 \alpha \rho\left(\alpha \tilde{\phi}^{2}+\tilde{\phi} \Gamma_{00} \tilde{\phi}\right)\right)}
\end{aligned}
$$

for some constants $c_{1}, \ldots, c_{7}$. We can write this as

$$
\square \tilde{\phi}=\rho^{n-1}\left(\rho B^{i j}(s, y, \tilde{\phi}, \partial \tilde{\phi}) \partial_{i} \partial_{j} \tilde{\phi}+T(s, y, \tilde{\phi}, \partial \tilde{\phi})\right), \quad \rho=s^{2}-|y|^{2},
$$

where $B^{i j}$ and $T$ are smooth functions of $(s, y), \tilde{\phi}$ and $\partial_{i} \tilde{\phi}$, for $i=0, \ldots, n$ vanishing to second respectively third order at $(\tilde{\phi}, \partial \tilde{\phi})=(0,0) ;\left|B^{i j}(s, y, \tilde{\phi}, \partial \tilde{\phi})\right| \leq C(|\tilde{\phi}|+$ $|\partial \tilde{\phi}|)^{2}$ and $|T(s, y, \tilde{\phi}, \partial \tilde{\phi})| \leq C(|\tilde{\phi}|+|\partial \tilde{\phi}|)^{3}$. The importance of (3.9) is that it is nonsingular at $\rho=0$. 
Having derived the transformation of the equation let us explain in what region it is applied. The transformation $\kappa$ maps the interior of the forward light cone $\{(s, y) ; s>|y|\}$ onto itself $\{(t, x) ; t>|x|\}$ and the boundary $\{(s, y) ; s=|y|\}$ to infinity. Its inverse $\kappa^{-1}(t, x)=\left(t /\left(t^{2}-|x|^{2}\right), x /\left(t^{2}-|x|^{2}\right)\right)=(s, y)$ maps forward light cones $\Lambda_{c}=\{(t, x) ; t-|x| \geq c>0\}$ into backward light cones $\tilde{\Lambda}_{c}=\{(s, y) ; s+$ $|y| \leq 1 / c, s-|y| \geq 0\}$ intersected with $\{(s, y) ; s \geq|y|\}$. The inverse is smooth on $\Lambda_{c}$ since $t^{2}-|x|^{2} \geq c^{2}>0$ there. Note also that hyperplanes $\tilde{\mathcal{H}}_{b}=\{(s, y) ; s=$ $1 / 2 b,|y| \leq 1 / 2 b\}$ are transformed to hyperboloids $\mathcal{H}_{b}=\left\{(t, x) ;(t-b)^{2}-|x|^{2}=b^{2}\right\}$. We will now use this transformation in $\Lambda_{c}$ which by the inverse is mapped to the compact region $\tilde{\Lambda}_{c}$ where we can use the standard local existence theorem for (3.9) to, after transforming back, obtain a global solution of our original equation (1.5) in $\Lambda_{c}$. Let us now explain how we can reduce it to a problem in $\Lambda_{c}$. First by scaling, $\phi_{a}(t, x)=\phi(a t, a x) / a$ is a solution of (1.5) if $\phi$ is; so we may assume that data (1.2) are supported in the set $\{x ;|x| \leq 1\}$. Second, we can translate the solution in the time direction so that initial conditions are attained when $t=a>1$. By the local existence theorem we have, if initial data when $t=a$ are sufficiently small, a solution $\phi$ to (1.5), for $|t-a| \leq a$, and it is as small as we wish there. Furthermore, by Huygens' principle the solution vanishes outside the forward light cone $\Lambda_{a-1}$, for $t \geq a$. We now want to show that the solution $\phi$ of (1.5) extends to a solution in all of $\Lambda_{a-1}$ for $t \geq a$ by showing that we have a local solution $\tilde{\phi}$ of (3.9) in the compact set $\tilde{\Lambda}_{a-1}$. We will now describe how to obtain initial conditions for (3.9). We pick a particular hyperboloid $\mathcal{H}_{b}$, where $b=(a-1 / a) / 2>0$, that intersects with the plane $t=a$ exactly when $|x|=1$, and that is transformed by the inverse of $\kappa$ to the plane $\tilde{\mathcal{H}}_{b}$. The intersection of $\mathcal{H}_{b}$ with the support of $\phi, \mathcal{H}_{b}^{1}=\left\{(t, x) \in \mathcal{H}_{b} ;|x| \leq 1\right\}$, is contained in the forward light cone $\Lambda_{a-1}$ intersected with the set where $t \leq a$. Hence we have a smooth solution $\phi$ on $\mathcal{H}_{b}^{1}$ which is as small as we wish there and which vanishes on $\mathcal{H}_{b} \backslash \mathcal{H}_{b}^{1}$. It follows that $\phi$ and its derivatives restricted to $\mathcal{H}_{b}$ are transformed onto smooth initial conditions for $\tilde{\phi}$ on $\tilde{\mathcal{H}}_{b}$, which are as small as we wish. The local existence theorem with these initial conditions on $\tilde{\mathcal{H}}_{b}$ gives us a smooth solution $\tilde{\phi}$ of $(3.9)$ in $\{(s, y) ; s \leq 1 /(2 b), s \geq|y|\}$ and hence in $\tilde{\Lambda}_{a-1}$ for $s \leq 1 /(2 b)$, if initial conditions on $\tilde{\mathcal{H}}_{b}$ are sufficiently small. Transforming back gives us a global smooth solution $\phi$ of (1.5) in $\Lambda_{a-1}$ and hence for all $t \geq a$. This concludes the proof.

\section{ACKNOWLEDGMENTS}

I would like to thank Demetri Christodoulou, Richard Hamilton and Jim Isenberg for stimulating discussions.

\section{REFERENCES}

[CC] Y. Choquet-Bruhat and D. Christodoulou, Existence of global solutions of the Yang-Mills, Higgs and spinor field equations in $3+1$ dimensions, Ann. Sci. École Norm. Sup. (4) 14 (1981), 481-506. MR 84c:81041

[C1] D. Christodoulou, Global solutions of nonlinear hyperbolic equations for small initial data, Comm. Pure Appl. Math. 39 (1986), 267-282. MR 87c:35111

[C2] - Oral communication, 1999.

[C3] Solutions globales des équations de Yang et Mills, C. R. Acad. Sci. Paris (Series A) 293 (1981), 139-141. MR 82k:58089

[CK1] D. Christodoulou and S. Klainerman, The nonlinear stability of Minkowski space-time, Princeton University Press, Princeton, NJ. 
[Ha1] R. Hamilton, Oral Communication, Oberwolfach, 1994.

[Ho1] J. Hoppe, Some classical solutions of relativistic membrane equations in 4-space-time dimensions., Phys. Lett. B 329, No. 1 (1994), 10-14. MR 95e:81180

[Hö1] L. Hörmander, $L^{1}, L^{\infty}$ estimates for the wave operator, Analyse Mathématique et Applications, Gauthier-Villars, Paris, 1988, pp. 211-234. MR 90e:35113

[Hö2] Lectures on nonlinear hyperbolic differential equations, Springer-Verlag, Berlin, 1997. MR 98e:35103

[HS1] G. Huisken and M. Struwe, Oral communication, 1999.

[JK1] F. John and S. Klainerman, Almost global existence to nonlinear wave equations in three space dimensions, Comm. Pure Appl. Math. 37 (1984), 443-455. MR 85k:35147

[K1] S. Klainerman, Uniform decay estimates and the Lorentz invariance of the classical wave equation, Comm. Pure Appl. Math. 38 (1985), 321-332. MR 86i:35091

[K2] , The null condition and global existence to nonlinear wave equations, Lectures in Applied Mathematics, Vol. 23, Amer. Math. Soc., Providence, RI, pp. 293-326. MR 87h:35217

[K3] , Global existence for nonlinear wave equations, Comm. Pure Appl. Math. 33 (1980), 43-101. MR 81b:35050

[K4] Long time behaviour of solutions to nonlinear wave equations, Proceedings of the International Congress of Mathematicians (Warsaw, 1983), PWN, Warsaw, 1984, pp. $1209-1215$

[LZ1] T. Li and Y. Zhou, Life-span of classical solutions to nonlinear wave equations in two space dimensions, J. Math. Pures et Appl. 73(3) (1994), 223-249. MR 95c:35174

[LZ2] T. Li and Y. Zhou, Life-span of classical solutions to fully nonlinear wave equations in two-space-dimensions II, J. Partial Differential Equations 6(1) (1993), 17-38. MR 95c:35175

[L1] H. Lindblad, On the lifespan of solutions of nonlinear wave equations with small initial data, Comm. Pure Appl. Math. 43 (1990), 445-472. MR 91i:35129

[L2] _ Global solutions of nonlinear wave equations, Comm. Pure Appl. Math. 45(9) (1992), 1063-1096. MR 94a:35080

Department of Mathematics, University of California at San Diego, La Jolla, CalIFORNIA 92093-0112

E-mail address: lindblad@math.ucsd.edu 